\title{
HUMAN NEUROPSIN GENE IN DEPRESSION
}

\author{
Kinga Bobińska ${ }^{1}$, Joanna Mossakowska-Wójcik ${ }^{1}$, Janusz Szemraj ${ }^{2}$, Piotr Gałecki ${ }^{1}$, \\ Marlena Zajączkowska ${ }^{1}$ \& Monika Talarowska ${ }^{1}$ \\ ${ }^{I}$ Department of Adult Psychiatry, Medical University of Lodz, Lodz, Poland \\ ${ }^{2}$ Department of Medical Biochemistry, Medical University of Lodz, Lodz, Poland
}

received: 7.7.2016;

revised: 11.4.2017;

accepted: 21.4 .2017

\section{SUMMARY}

Background: Neuropsin (NP, kallikrein 8, KLK8) - a kallikrein gene-related (KLK) endoprotease - plays a key role in neuroplasticity processes, since intracellular signal cascades and regulation of gene expression are engaged in long-term synaptic plasticity. The main aim of this paper is to compare expression of the human neuropsin gene on the mRNA level in a group of patients diagnosed with depression and in a group of healthy subjects who have never been treated psychiatrically.

Subjects and methods: 291 people, aged 18-67, were qualified to participate in the experiment: major recurrent depression group (MRD) and the control group (CG). Designations were carried out for the human NP gene (hNP).

Results: For hNP gene expression at the mRNA level was higher in patients with depression than in the CG $(p<0.005)$. A Spearman's rank correlation analysis did not reveal any statistically significant relationship between the intensity of the disease measured using the HDRS scale and expression on the mRNA level for the hNP gene. Expression for the hNP gene in the entire group analysed increased with age of the examined individuals $(p<0.005)$.

Conclusion: Expression of the hNP gene on the mRNA level, evaluated based on peripheral blood, is significantly higher in the patients with MRD than in the healthy subjects.

Key words: major recurrent depression - human neuropsin - kallikrein

$* * * * *$

\section{INTRODUCTION}

Neuropsin (NP, kallikrein 8, KLK8) - a kallikrein gene-related (KLK) endoprotease - plays a key role in neuroplasticity processes, since intracellular signal cascades and regulation of gene expression are engaged in long-term synaptic plasticity (Ziemiańska et al. 2012). Enzymatically active neuropsin is necessary to establish the early phase of long-term potentiation (E-LTP, early LTP), while neuropsin deficiency completely impairs the early phase of LTP, leading to the absence of late associativity. These phenomena are extremely significant in the process of acquiring new skills (Lu 2009, Shiosaka et al. 2011) in both humans and animals (Tamura et al. 2006).

Neuropsin is a type of serine proteases, which are involved in extracellular proteolysis. Serine proteases belong to the class of hydrolases, subclass of proteases, and are in charge of selective catalysis of peptide bond hydrolysis. They constitute one third of the proteases found in nature (Hedstrom 2002).

The human NP (hNP) gene was cloned by Yoshida et al. (1998) and then localized to chromosome 19q13.3-q13.4. It consists of six exons, with the first exon being nontranslational. Four alternative splicing variants have been identified. The regular form is called type 1; type 2 contains a 135-bp insertion of 5' upstream region of exon 3 . Type 2 is a hominoid-specific splicing form and is expressed as abundantly as the type 1 in the human brain (Izumi et al. 2008).

As early as in 1999, a team led by Mitsui et al. (1999) confirmed dominant expression of type 1 neuropsin in the pancreas and of type 2 neuropsin in the brain of adult individuals, mainly within the area of the hippocampus. According to the cited authors, these proportions are completely different during embryonic life. Similar expression of the two types of neuropsin is observed in the brain of a developing foetus and within the vicinity of the placenta (Mitsui et al. 1998). Type 2 neuropsin is abundantly expressed in human embryonic stem cells and in embryo brain samples, suggesting a potential role in embryogenesis (Lu et al. 2009). The data presented above allow to draw a conclusion about the significant role of type 2 neuropsin in neuroplasticity processes taking place in an adult brain, although both types pf neuropsin may be also important in the development of the nervous system.

Neuropsin expression takes places both extracellularly and inside neurons within the area of the hippocampus (Ziemiańska et al. 2012). Neuropsin inhibitors (SPI3 and MUG I) are expressed by pyramidal neurons of the hippocampus (Kato et al. 2001). Various forms of electrophysiological stimulation (kindling, LTP, stress) increase neuropsin expression within the hippocampus and in many other regions of the brain (e.g. neocortex, amygdala) (10). The precursor form of neuropsin is secreted to the extracellular space and is activated during LTP in an NMDA receptor-dependent manner. Activation of neuropsin occurs by cleavage of a 4amino acid peptide (QGSK) after a signal is received from downstream of the NMDA receptor (MatsumotoMiyai et al. 2003, Ishikawa et al. 2011). 
Neuropsin is mainly engaged in the early stage of LTP and in the process of synaptogenesis (Ziemiańska et al. 2012), while its deficiency reduces the seizure threshold (Davies et al. 2001). Decreased expression of hNP has been confirmed not only in association with deteriorated cognitive functioning, but also in the people affected by brain dysfunctions (among others suffering from multiple sclerosis, SM) (Dutra et al. 2013, Harris et al. 2010).

The hNP gene maps to chromosome $19 q 13$ - a region reportedly linked to schizophrenia and bipolar disorders (Izumi et al. 2008). Increased expression of $\mathrm{hNP}$ in the human brain is observed in the regions that are engaged in the course of depression: hippocampus, amygdala, olfactory bulb, and prefrontal cortex (Horii et al. 2008). Particularly high expression of hNP on the mRNA level has been confirmed in the pyramidal neurons of the hippocampus, within the CA1-CA3 fields (Chen et al. 1995), which are associated with the phenomenon of neuroplasticity.

The main aim of this paper is to compare expression of the human neuropsin gene on the mRNA level in a group of patients diagnosed with depression and in a group of healthy subjects who have never been treated psychiatrically. The authors formulated a hypothesis regarding significant differences in hNP expression between the examined groups. Based on a review of available literature, assumptions were made about dominating $\mathrm{hNP}$ expression in the healthy individuals as compared to the patients with major recurrent depression (MRD).

\section{SUBJECTS AND METHODS}

\section{Subjects}

291 people, aged 18-67 $(M=41.13, S D=13.7)$, were qualified to participate in the experiment. The participants were divided into two groups: the people suffering from depressive disorders (major recurrent depression, MRD) - 186 people, and the control group (CG) - 105 people. The individuals taking part in the experiment were native Poles from central Poland (not related).

The patients were selected on the basis of the inclusion criteria for ED and $\mathrm{rDD}$ specified in ICD-10 (F32.0-7.32.2, F33.0-F33.8) (WHO 2015) and DEM-5 (APA 2013). All the subjects were examined during their hospitalisation and no symptoms of concurrent somatic diseases or axis I and II disorders other than depressive episodes were diagnosed in them. Inflammatory or autoimmune disorders, central nervous system traumas and unwillingness to give informed consent were additional exclusion criteria. A case history was obtained from each patient using the standardised Composite International Diagnostic Interview (CIDI) (Patten 1997) prior to the start of the experiment). The patients from the rDD group were qualified for the study during the process of pharmacological therapy modification. They were treated with agents from the group of selective serotonin reuptake inhibitors (SSRI).

The CG comprised healthy subjects without any familial cases of mental disorders. The group of healthy controls was composed of community volunteers who were qualified for the study based on the criteria of the psychiatric CIDI interview (Patten 1997). Individuals suffering from other psychiatric diseases, axis I and II disorders, neurological disorders, or diagnosed with substance abuse or dependence, were excluded from the experiment.

The control subjects or depressed patients were not treated with the use of the drugs known to influence lipid metabolism, immune responses or endocrine functions. The control subjects had not been taking any medications for at least 2 months prior to blood sampling. None of the healthy participants was a drinker or heavy smoker, and none had ever taken psychotropic drugs.

\section{Methods}

Depression severity was evaluated and classified using the 21-item Hamilton Depression Rating Scale (HDRS) (Hamilton 1960). Intensity levels of depressive symptoms were measured with the use of the grades presented in the study conducted by Demyttenaere and De Fruyt (2003). Each patient was examined by the same specialist (the same psychiatrist performed the HDRS test).

The analysed blood was collected from the examined individuals (both in the experimental and comparative group) once - on the day of enrolment. The assessment of depression symptoms intensification based on the HDRS scale was conducted in the experimental group twice - at the beginning of the therapy and after 8 weeks.

Peripheral blood was used as a material in the genotype study (in volumes of $5 \mathrm{ml}$ on EDTA).

\section{Total RNA isolation}

Total RNA isolation from the patients' blood samples using an RNA extraction reagent, TRIZOL (Invitrogen Life Technologies), according to the standard acid-guanidinium-phenol-chlorophorm method was performed based on the modified Chomczyński method. Absorbance of isolated RNA was measured using a spectrophotometer (Picodrop) at $\lambda=260 \mathrm{~nm}$ in order to determine total RNA concentration. Isolated RNA was stored at a temperature of $-70^{\circ} \mathrm{C}$.

\section{Quality analysis of isolated RNA}

The quality of total RNA was verified with the Agilent RNA 6000 Nano Kit (Agilent Technologies) in accordance with the manufacturer's recommendations. 1 $\mu 1$ of RNA 6000 Nano dye was added to a test tube containing $65 \mu \mathrm{l}$ of Agilent RNA 6000 Nano gel matrix and then centrifuged (10 minutes, $13000 \mathrm{xg}$ ). The gelfluorescent dye mixture was applied on the surface of a 
Nano chip placed in a workstation. Then, $5 \mu \mathrm{l}$ of RNA Nano marker was added to selected pits. Isolated samples of RNA and RNA size marker were subject to denaturation $\left(2\right.$ minutes, $\left.70^{\circ} \mathrm{C}\right)$, and then $1 \mu \mathrm{l}$ of the sample was pipetted to selected pits of the Nano chip and mixed (1 minute, $2400 \mathrm{rpm}$ ). The quality of isolated RNA was checked using the 2100 Bioanalyzer (Agilent Technologies). The level of degradation of total RNA was determined with the use of an electrophoretogram and RIN values recorded. Only the samples with RIN value $>7$ were subject to further analysis.

\section{RT-PCR reverse transcription}

An RT reaction was carried out with the TaqMan ${ }^{\circledR}$ RNA Reverse Transcription Kit (Applied Biosystems) based on the manufacturer's recommendations, using Hs01012737_m1, Hs04194366_g1 probes, specific respectively for the hNP (human neuropsin gene) and RPL13A genes, delivered by Applied Biosystems. The samples were incubated $\left(30\right.$ minutes at $16^{\circ} \mathrm{C}$ and 30 minutes at $42^{\circ} \mathrm{C}$ ) in a thermocycler (Biometra). Reverse transcriptase was inactivated $\left(5\right.$ minutes at $\left.85^{\circ} \mathrm{C}\right)$ and the obtained cDNA was stored at a temperature of $20^{\circ} \mathrm{C}$.

\section{Real-Time PCR reaction}

Real-Time PCR reaction was conducted using the TaqMan ${ }^{\circledR}$ Universal PCR Master Mix, No UNG (Applied Biosystems) according to the protocol provided by the manufacturer. The reaction mixture ratio was presented in the table. To calculate relative expression of miRNA genes, the $\mathrm{Ct}$ comparative method was used (Livak and Schmittgen 2001; Schmittgen and Livak 2008). The level of hNP gene expression in blood was normalised in relation to the RPL13A reference gene.

Each target probe was amplified in a separate 96well plate. All samples were incubated at $50^{\circ} \mathrm{C}$ for 2 minutes and at $95^{\circ} \mathrm{C}$ for 10 minutes, and then cycled at $95^{\circ} \mathrm{C}$ for 30 seconds, at $60^{\circ} \mathrm{C}$ for 30 seconds and at $72^{\circ} \mathrm{C}$ for 1 minute; 40 cycles were performed in total. Fluorescence emission data were captured and mRNA levels were quantified using the critical threshold $(\mathrm{Ct})$ value. Analyses were performed with ABI Prism 7000 (SDS Software). Controls without RT and with no template cDNA were performed with each assay. Relative gene expression levels were obtained using the $\Delta \Delta \mathrm{Ct}$ standard 2- $\Delta \Delta \mathrm{ct}$ calculations and expressed as a fold change of the control sample (Livak \& Schmittgen 2001, Schmittgen \& Livak 2008). Amplification specific transcripts were further confirmed by obtaining melting curve profiles.

\section{Statistical Analyses}

A statistical analysis of the material was performed with the use of both descriptive and inferential statistics. A two-tailed critical region was employed in the testing of the statistical hypothesis.
The qualitative characteristics of the groups of affected subjects and healthy controls were expressed as frequencies and shown as percentages. An arithmetical mean $(\mathrm{M})$ and median (Me) were calculated in order to characterise the average values of quantitative features. Statistical dispersion measures included the values between the minimum and the maximum, and standard deviation (SD).

Distributions were measured with the use of the Shapiro-Wilk test. The following tests were applied in the comparison of nonparametric variables in the test groups: the Pearson $\chi^{2}$ for qualitative variables, the Wilcoxon signed-rank test for two related groups for quantitative variables, and the Mann-Whitney U test for two independent groups to determine the coincidence of distributions. Spearman's R rank order correlation coefficients were estimated, the aim of which was to evaluate the relationships between the analysed variables. Statistical significance was defined as $\mathrm{p}<0.05$ (Kirkwood \& Sterne 2003). All the analyses were conducted using STATISTICA PL, version 10.

\section{Ethics}

The individuals taking part in the experiment were chosen for the study group at random without replacement sampling. Participation in the study was voluntary; any personal data and results were kept confidential. Before making a decision to participate in the study, the subjects were informed of the purpose, assured of the voluntary character of the experiment and guaranteed that their personal data would be kept in secret. Written informed consent was given in accordance with the study protocol, approved by the Bioethical Committee of the Medical University of Lodz (No. RNN/272/15/KE).

\section{RESULTS}

The social and demographic characteristics of the examined individuals and the information regarding the course of the disease are presented in table 1.

No significant differences between the analysed groups were found in terms of $\operatorname{sex}\left(\chi^{2}=4.84, p=0.27\right)$; the only difference referred to age of the examined individuals (Table 1).

Significant differences were observed in the MRD group in terms of the intensity of depression symptoms measured at the beginning of a pharmacotherapy and after 8 weeks. A significant improvement of the patients' mental status was achieved (Table 1).

Significant differences were confirmed in the expression of hNP on the mRNA level between the individuals from the control group and the affected diagnosed with MRD $(Z=5.86, p<0.005)$, respectively for the CG group: $M=0.142(S D=0.03)$ and for the rDD group: $M=0.187(S D=0.07)$. Expression of $\mathrm{hNP}$ on the mRNA level for the entire group analysed reached the following value: $M=0.171 \quad(S D=0.06)$. The obtained results can be found in figure 1 . 
Table 1. Comparison of the study groups in terms of age, sex and the course of depression

\begin{tabular}{|c|c|c|c|c|c|}
\hline & \multicolumn{2}{|c|}{$\operatorname{MRD}(\mathrm{N}=186)$} & \multicolumn{2}{|c|}{$\mathrm{CG}(\mathrm{N}=105)$} & \multirow{2}{*}{$\begin{array}{c}\text { Total }(\mathrm{N}=291) \\
\mathrm{N}(\%)\end{array}$} \\
\hline & $\mathrm{N}$ & $\%$ & $\mathrm{~N}$ & $\%$ & \\
\hline \multicolumn{6}{|l|}{ Sex } \\
\hline Female & 117 & 62.91 & 69 & 65.71 & 63.92 \\
\hline Male & 69 & 37.09 & 36 & 34.29 & 36.08 \\
\hline Variable & $\mathrm{M}$ & $\mathrm{SD}$ & $\mathrm{M}$ & $\mathrm{SD}$ & Mann-Whitney U test \\
\hline Age & 48.03 & 10.99 & 28.89 & 8.68 & $<0.005^{*}$ \\
\hline & & & & & Wilcoxon signed-rank te \\
\hline HDRS-I & 23.27 & 6.51 & - & - & \multirow{2}{*}{$<0.005^{*}$} \\
\hline HDRS-II & 6.63 & 4.31 & - & - & \\
\hline Duration of disease (in years) & 6.31 & 4.23 & - & - & - \\
\hline
\end{tabular}

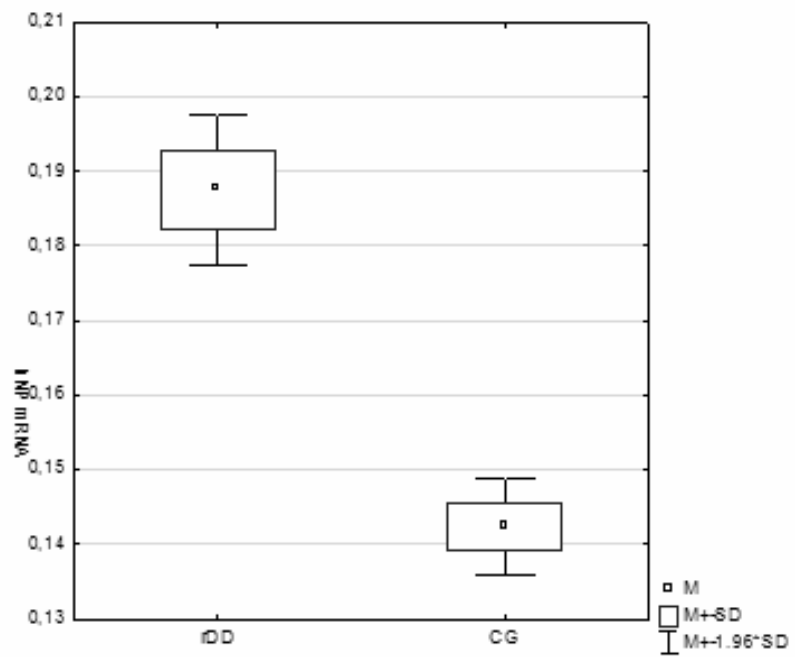

$\mathrm{CG}$ - control group; $\mathrm{n}$ - number of samples; $\mathrm{MRD}$ - major recurrent depression; $\mathrm{M}$ - mean; $\mathrm{SD}$ - standard deviation

Figure 1. Expression of hNP on the mRNA level in the examined groups

A Spearman's rank correlation analysis did not reveal any statistically significant relationship between the intensity of the disease measured using the HDRS scale and expression on the mRNA level for the hNP gene.

Expression for the hNP gene increased with age in the entire group of the examined individuals $(R=0.211$, $p<0.005)$. No statistical significance was observed when the two groups were analysed separately $(R=-0.056$, $p=0.443$ for the MRD group; $R=-0.076, p=0.435$ for the $\mathrm{CG}$ group). However, the nature of the analysed interrelation was completely different, i.e. expression of hNP on the mRNA level decreased with age of the examined individuals.

\section{DISCUSSION}

The hypothesis formulated by the authors in the introduction was not confirmed. The results obtained indicate increased hNP expression on the mRNA level in the individuals treated due to depression symptoms as compared to the healthy subjects. Our team analysed hNP expression in the group of patients with MRD; due to the fact that no experiment of this kind has ever been conducted so far, we did not have an opportunity to compare our results with the reports from other scientific centres. As regards the reasons that could explain the results obtained, we may only base on contemporary theories which describe the aetiology of depressive disorders. One of them is the inflammatory theory of depression, in the light of which the research results obtained by Viel and Buck (2011) seem very interesting. The Kallikrein-Kinin System (KKS) has been associated to inflammatory and immunogenic responses in the peripheral and central nervous system by the activation of two receptors, namely $\mathrm{B} 1$ receptor and $\mathrm{B} 2$ receptor. The $\mathrm{B} 1$ receptor is absent or under-expressed in physiological conditions, being up-regulated during tissue injury or in the presence of cytokines. The B2 receptor is constitutive and mediates most of the biological effects of kinins (Viel \& Buck 2011). Increased expression of $\mathrm{hNP}$ in people with depression, as a defense reaction of the organism against disease recurrence, is only a hypothesis which can be verified in subsequent studies.

Interestingly, the results recorded by our team indicate an increase of hNP expression on the mRNA level with age of the examined patients for the entire analysed group $(N=291)$ and a drop in the expression of the gene when analyses are carried out separately for the two groups. The results recorded may be affected by the average age of the examined group $(M=41.13, S D=13.7$, i.e. middle adulthood). Reports from available studies on NP expression in association with age of participants are not unambiguous. According to Konar and Thkur (2015), NP expression gradually decreases in the cerebral cortex during aging. A sharp rise is observed in both the olfactory bulb and the hippocampus in adults, followed by a decline in old age. However, according to Dukic et al. (2016), serum concentrations of NP do not differ between Alzheimer Disease (AD), Vascular Dementia (VAD) and controls with and without mild cognitive impairment. 
It seems that further studies regarding the significance of hNP in the aetiology and course of rDD should be aimed at finding out their impact on the cognitive efficiency of the affected (Lu et al. 2009, Tsilibary et al. 2014) and on their emotional functioning (Horii et al. (2008) found increased anxiety in neuropsin-deficient mice, suggesting the involvement of this protease in emotional responses).

\section{CONCLUSIONS}

Expression of the hNP gene on the mRNA level, evaluated based on peripheral blood, is significantly higher in the patients with MRD than in the healthy subjects.

\section{Limitations}

Fairly young age of the examined individuals in both groups, which may be the reason for the differences in hNP expression.

Additionally, the difference in mean age of the examined individuals may be significant for the analysis of the obtained results.

\section{Acknowledgements:}

Financial support: no.: 503/5-062-02/503-51-004.

All authors have approved the final article.

Conflict of interest: None to declare.

\section{Contribution of individual authors:}

Kinga Bobińska: study design, data collection, data interpretation, manuscript preparation, literature search;

Joanna Mossakowska-Wójcik: revision preparation, manuscript preparatiaon, literature search;

Janusz Szemraj: genetic analysis;

Piotr Gałecki: study design, data interpretation, critical review;

Marlena Zajączkowska: statistical analysis;

Monika Talarowska: data interpretation, manuscript preparation, literature search.

\section{References}

1. APA: Diagnostic and Statistical Manual of Mental Disorders fifth Edition. APA (US), 2013.

2. Chen ZL, Yoshida S, Kato K, Momota Y, Suzuki J, Tanaka $T$ et al.: Expression and activity-dependent changes of a novel limbic-serine protease gene in the hippocampus. $J$ Neurosci 1995; 15:5088-97.

3. Davies B, Kearns IR, Ure J, Davies CH, Lathe R: Loss of hippocampal serine protease BSP1/neuropsin predisposes to global seizure activity. J Neurosci 2001; 21:6993-7000.

4. Demyttenaere K, De Fruyt J: Getting what you ask for: on the selectivity of depression rating scales. Psychothery Psychosom 2003; 72:61-70.
5. Dukic L, Simundic AM, Martinic-Popovic I, Kackov S, Diamandis A, Begcevic I, et al.: The role of human kallikrein 6, clusterin and adiponectin as potential blood biomarkers of dementia. Clin Biochem 2016; 49:213-8.

6. Dutra RC, Moreira EL, Alberti TB, Marcon R, Prediger $R D$, Calixto JB: Spatial reference memory deficits precede motor dysfunction in an experimental autoimmune encephalomyelitis model: the role of kallikrein-kinin system. Brain Behav Immun 2013; 33:90-101.

7. Hamilton M: A rating scale for depression. J Neurol Neurosurg Psychiatry 1960; 23:56-62.

8. Harada A, Shiosaka S, Ishikawa Y, Komai S: Acute stress increases neuropsin $m R N A$ expression in the mouse hippocampus through the glucocorticoid pathway. Neuroscience Lett 2008; 436:273-7.

9. Harris VK, Diamanduros A, Good P, Zakin E, Chalivendra V, Sadiq SA: Bri2-23 is a potential cerebrospinal fluid biomarker in multiple sclerosis. Neurobiol Dis 2010; 40:331-9.

10. Hedstrom L: Serine protease mechanism and specificity. Chem Rev 2002; 102:4501-24.

11. Horii Y, Yamasaki N, Miyakawa T, Shiosaka S: Increased anxiety-like behavior in neuropsin (kallikrein-related peptidase 8) gene-deficient mice. Behav Neurosci 2008; 122:498-504.

12. Ishikawa Y, Tamura H, Shiosaka S: Diversity of neuropsin (KLK8)-dependent synaptic associativity in the hippocampal pyramidal neuron. J Physiol 2011; 589(Pt 14):3559-73.

13. Izumi A, Iijima Y, Noguchi H, Numakawa T, Okada T, Hori $H$ et al.: Genetic variations of human neuropsin gene and psychiatric disorders: polymorphism screening and possible association with bipolar disorder and cognitive functions. Neuropsychopharmacology 2008; 33:3237-45.

14. Kato K, Kishi T, Kamachi T, Akisada M, Oka T, Midorikawa $R$ et al.: Serine proteinase inhibitor 3 and murinoglobulin $I$ are potent inhibitors of neuropsin in adult mouse brain. J Biol Chem 2001; 276:14562-71.

15. Kirkwood B \& Sterne J: Essential medical statistics, 2nd edition. Wiley-Bleckwell, 2003.

16. Konar A \& Thakur MK: Neuropsin Expression Correlates with Dendritic Marker MAP2c Level in Different Brain Regions of Aging Mice. Mol Neurobiol 2015; 51:1130-8.

17. Livak $K$ \& Schmittgen TD: Analysis of relative gene expression data using real-time quantitative PCR and the 2- $\triangle 4 C$ T method. Methods 2001; 25:402-8.

18. Lu ZX, Huang $Q, S u B$ : Functional characterization of the human-specific (type II) form of kallikrein 8, a gene involved in learning and memory. Cell Res 2009; 19:259-67.

19. Matsumoto-Miyai K, Ninomiya A, Yamasaki H, Tamura H, Nakamura Y, Shiosaka S: NMDA-dependent proteolysis of presynaptic adhesion molecule L1 in the hippocampus by neuropsin. J Neurosci 2003; 23:7727-36.

20. Mitsui S, Tsuruoka N, Yamashiro $K$, Nakazato $H$, Yamaguchi N: A novel form of human neuropsin, a brainrelated serine protease, is generated by alternative splicing and is expressed preferentially in human adult brain. Eur J Biochem 1999; 260:627-34.

21. Patten S: Performance of the Composite International Diagnostic Interview Short Form for major depression in community and clinical samples. Chron Dis Can 1997; $3: 18-24$. 
22. Schmittgen TD \& Livak KJ: Analyzing real-time PCR data by the comparative CT method. Nat Protoc 2008; 3:1101-8.

23. Shiosaka $S$ \& Ishikawa Y: Neuropsin - a possible modulator of synaptic plasticity. J Chem Neuroanat 2011; 42:24-9.

24. Tamura H, Ishikawa Y, Hino N, Maeda M, Yoshida S, Kaku $S$ et al.: Neuropsin is essential for early processes of memory acquisition and Schaffer collateral long-term potentiation in adult mouse hippocampus in vivo. J Physiol 2006; 570:541-51.

25. Tsilibary E, Tzinia A, Radenovic L, Stamenkovic V, Lebitko T, Mucha M et al.: Neural ECM proteases in learning and synaptic plasticity. Prog Brain Res 2014; 214:135-57.
26. Viel TA \& Buck HS: Kallikrein-kinin system mediated inflammation in Alzheimer's disease in vivo. Curr Alzheimer Res 2011; 8:59-66.

27. WHO: International Statistical Classification of Diseases and Related Health Problems 10th Revision (ICD-10). World Health Organization, Genewa, 2015.

28. Yoshida S, Taniguchi M, Hirata A, Shiosaka S: Sequence analysis and expression of human neuropsin $c D N A$ and gene. Gene 1998; 213:9-16.

29. Ziemiańska K, Konopka A, Wilczyński GM: The role of extracellular proteolysis in synaptic plasticity of the central nervous system. Advances in Hygiene and Experimental Medicine (Online) 2012; 66:959-75.

\section{Correspondence:}

Prof. Monika Talarowska, MD, PhD

Department of Adult Psychiatry, Medical University of Lodz Aleksandrowska 159, 91-229, Lodz, Poland

E-mail: talarowskamonika@wp.pl 